\title{
Meteoric Layers in Planetary Atmospheres
}

\author{
J.G. Molina-Cuberos · J.J. López-Moreno · F. Arnold
}

Received: 11 December 2007 / Accepted: 6 March 2008 / Published online: 22 April 2008

(C) Springer Science+Business Media B.V. 2008

\begin{abstract}
Metallic ions coming from the ablation of extraterrestrial dust, play a significant role in the distribution of ions in the Earth's ionosphere. Ions of magnesium and iron, and to a lesser extent, sodium, aluminium, calcium and nickel, are a permanent feature of the lower E-region. The presence of interplanetary dust at long distances from the Sun has been confirmed by the measurements obtained by several spacecrafts. As on Earth, the flux of interplanetary meteoroids can affect the ionospheric structure of other planets. The electron density of many planets show multiple narrow layers below the main ionospheric peak which are similar, in magnitude, to the upper ones. These layers could be due to long-lived metallic ions supplied by interplanetary dust and/or their satellites. In the case of Mars, the presence of a non-permanent ionospheric layer at altitudes ranging from 65 to $110 \mathrm{~km}$ has been confirmed and the ion $\mathrm{Mg}^{+} \cdot \mathrm{CO}_{2}$ identified. Here we present a review of the present status of observed low ionospheric layers in Venus, Mars, Jupiter, Saturn and Neptune together with meteoroid based models to explain the observations. Meteoroids could also affect the ionospheric structure of Titan, the largest Saturnian moon, and produce an ionospheric layer at around $700 \mathrm{~km}$ that could be investigated by Cassini.
\end{abstract}

Keywords Meteoroids · Ionosphere $\cdot$ Planetary atmosphere

J.G. Molina-Cuberos $(\bowtie)$

Depto. Física, Universidad de Murcia, Campus Espinardo, 30100, Murcia, Spain

e-mail: gregomc@um.es

\section{J.J. López-Moreno}

Instituto Astrofísica de Andalucía, CSIC, P.O. Box 3004, 18080, Granada, Spain

e-mail: lopez@iaa.es

\section{F. Arnold}

Atmospheric Physics Division, Max-Plank Institut for Nuclear Physics (MPIK), P.O. Box 10399980, 69029, Heidelberg, Germany

e-mail: frank.arnold@mpi-hd.mpg.de 


\section{Introduction}

The ablation of a continuous flux of extraterrestrial dust in the atmosphere gives rise to permanent layers of free neutral and ionized metal atoms in the $80-110 \mathrm{~km}$ altitude range. From the initial ground based observations of sodium at the end of the 1930s (Chapman 1938) to the in-situ measurements of metal ions by rocket-borne mass spectrometers (Grebowsky et al. 1998), the density profiles of metallic species as well as their latitude and temporal variability has been established. Magnesium and iron are the most abundant metal species in the atmosphere; others like sodium, aluminium, calcium and nickel are also present in a concentration, at least, one order of magnitude lower. The relative abundance of metal species in the atmosphere is roughly equal to the one exhibited in carbonaceous chondrites (Mason 1971).

In addition to general background of extraterrestrial dust, meteor showers, that are produced when the Earth crosses the dust stream left along a comet orbit, can increase the concentration of metals during short periods of time. The net mass influx to the Earth from each meteor shower is only a small fraction of the total yearly influx from the sporadic background (Hughes 1978). However, an increase by a factor of 2-3 in metallic concentration has been found during such events. The increase can be as much as one order of magnitude during a strong meteor shower (Kopp 1997; Grebowsky et al. 1998), which is often large enough to be manifested as a peak in the total ion and electron density profiles. Figure 1 shows two examples of the distribution of positive ions and electrons in the terrestrial atmosphere, where the increase in the metallic ion concentrations during the Perseid meteor shower is highlighted.

Dust detectors on board several space missions have observed that meteoroids are distributed through the whole interplanetary medium in the Solar System. Meteoroids can thus affect the atmospheric structure of other planets. In this paper we search for evidence of meteoroid layers in the atmospheres of extraterrestrial planets and we review the present knowledge of meteoroid modeling in the Solar System.
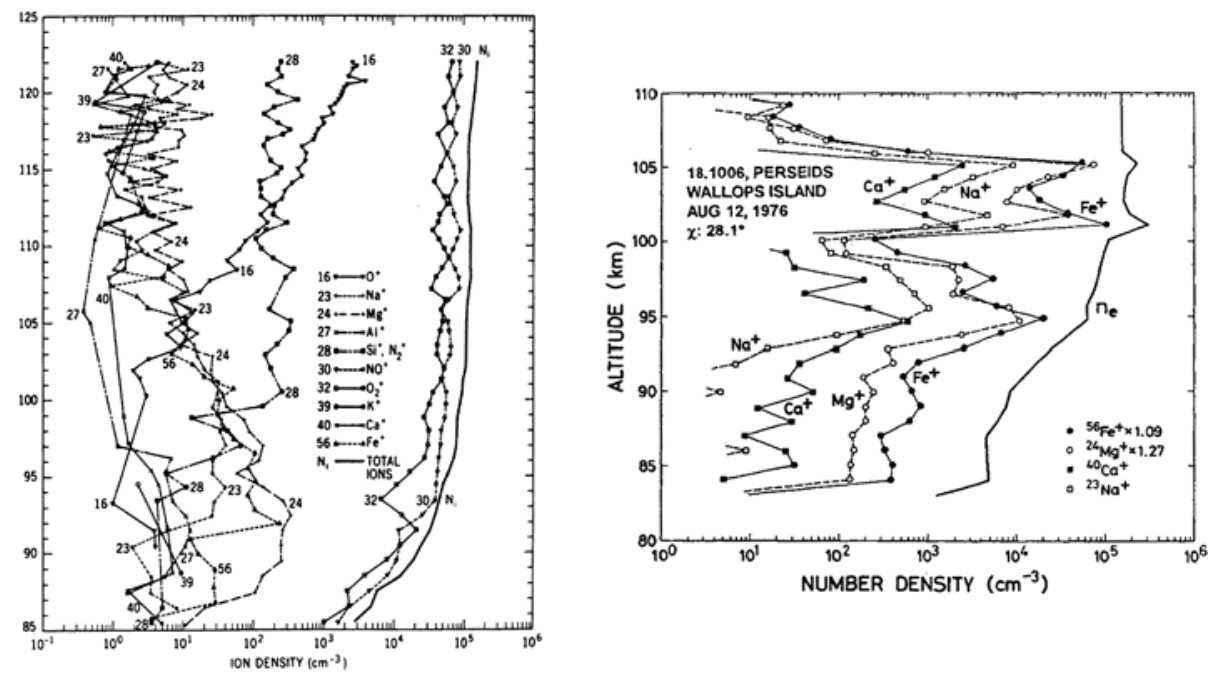

Fig. 1 Observed distribution of positive ion species in the terrestrial ionosphere during the following conditions: (left) daytime over Thumba (India) (Aikin and Goldbert 1973); (right) Perseid meteor shower on August 12, 1976, above Wallops Islands (Kopp 1997) 


\section{Evidence of Meteoroid Layers through the Solar System}

Planetary ionospheres have been sounded by radio occultation techniques since the beginning of the 1960s; the measurements show a daytime ionosphere with a major peak mainly produced by solar radiation and photoelectrons. Below the main peak, one or more secondary ionospheric layers have been found. Examples of such layers have been found at Venus by Pioneer Venus (Kliore et al. 1979) and Mars by Mariner IV (Fjeldbo et al. 1966), Mars 4 and 5 (Savich et al. 1976) and Mars Express (Pätzold et al. 2005) among others, see Fig. 2. The number of missions to the external planets is not so comprehensive, Galileo (Hinson et al. 1997) and Voyager (Hinson et al. 1998) found evidence of such layers in the Jovian atmosphere, Cassini (Nagy et al. 2006) in the atmosphere of Saturn and Voyager at Uranus (Strobel et al. 1991) and Neptune (Lyons 1995), see Fig. 3.

\subsection{Venus}

The electron density profile measured by Pioneer Venus in the nightside ionosphere shows low altitude layers below the main ambient ionospheric layer, see Fig. 2. The altitude of the main peak is located at $142.2 \pm 4.1 \mathrm{~km}$, very close to the main peak of the dayside terminator ionosphere. The peak density is characterized by a great variability, with a magnitude ranging from $23 \times 10^{3}$ to $40 \times 10^{3} \mathrm{~cm}^{-3}$ (Kliore et al. 1979). A double-peak structure appears during two closely spaced orbits, 55 and 57, and on orbit 57 the structure appeared in both the entry and exit measurements. It seems that the appearance of such a double-peak structure is a relatively rare temporal phenomenon. The altitude of the layer, $\sim 120 \mathrm{~km}$, is in agreement with the maximum for meteoroid ablation. Other ionization sources, such us direct impact ionization by electron precipitation or protons into the nightside, could also explain the nature of the lower ionospheric layer.

\subsection{Mars}

The atmosphere of Mars has been sounded in more detail than the rest of the extraterrestrial solar system planets, and its ionosphere presents the strongest evidences of metallic layers. The magnesium ion $\mathrm{Mg}^{+} \cdot \mathrm{CO}_{2}$ has even been identified as a constituent of the Martian ionosphere (Aikin and Maguire 2005). The daytime ionosphere is well characterized by a main layer produced by solar radiation at an altitude of $140 \mathrm{~km}$ with a number density of some teens of thousands electrons per cubic centimeter. Mariner IV found a secondary layer one order of magnitude lower at around $100 \mathrm{~km}$, below the main photoionospheric peak (Fjeldbo et al. 1966). Some years later, the soviet Mars 4 and 5 (Savich et al. 1976) found a layer at around $80 \mathrm{~km}$ during nightime similar, in magnitude, to the daytime one. Mars Express confirmed the existence of a sporadic layer between 65 and $110 \mathrm{~km}$ in altitude in 10 of 120 ionospheric electron concentration profiles (Pätzold et al. 2005). Figure 2 shows the measurements developed by Mars 4 and 5 (left) and Mars Express (right). The occurrence of the daytime layer was not limited to specific times of the day or locations, and part of it is hidden in the lower portion of the upper one (Pätzold et al. 2005). Mars Express did not find such layers in the 20 ionospheric observations at night, all of which were at high southern latitudes during winter. The observations are too limited to exclude the occurrence of a layer at night (Pätzold et al. 2005). Theoretical models considering meteoroids ablation indicate that the altitude and magnitude of the observed layers can be explained by long lived metallic ions deposited by meteoroids (Pesnell and Grebowsky 2000; Molina-Cuberos et al. 2003). 

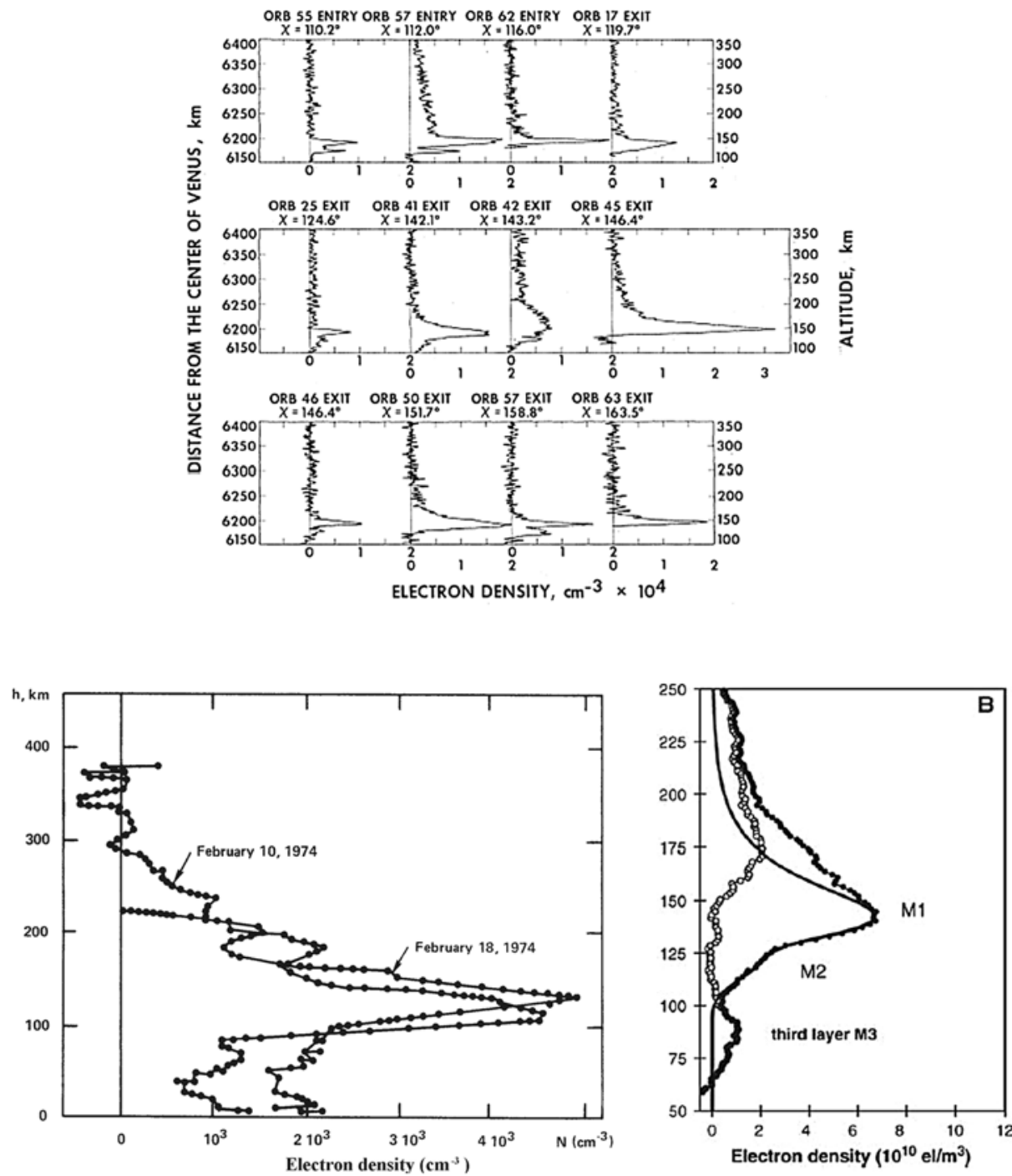

Fig. 2 Top electron concentration profiles in the nightside ionosphere of Venus measured by Pioneer Venus. From A.J. Kliore et al., Science 205:99-102 (July 6 1979). Reprinted with permission from AAAS. Lower-left distribution of electron concentration in the nighttime ionosphere of Mars measured by Mars 4 and 5 (Savich et al. 1976). Lower-right electron concentration in the Martian ionosphere observed by Mars Express (solid circles) and after subtracting a Chapman ionization model (open circles). From M. Pätzold et al., Science 310:837-839 (2005). Reprinted with permission from AAAS

\subsection{Jupiter}

The Voyager 2 fly-by provided most of the information about the lower ionospheric structure of Jupiter (Hinson et al. 1998). The electron concentration profile obtained during the egress contains two distinct layers: one is centred near $1000 \mathrm{~km}$ (relative to the 1 bar altitude) with a peak number density of $46 \times 10^{4} \mathrm{~cm}^{-3}$ and the structure of the other is more complex, see Fig. 3. It is formed by a group of fine layers situated between 300 and $500 \mathrm{~km}$ 

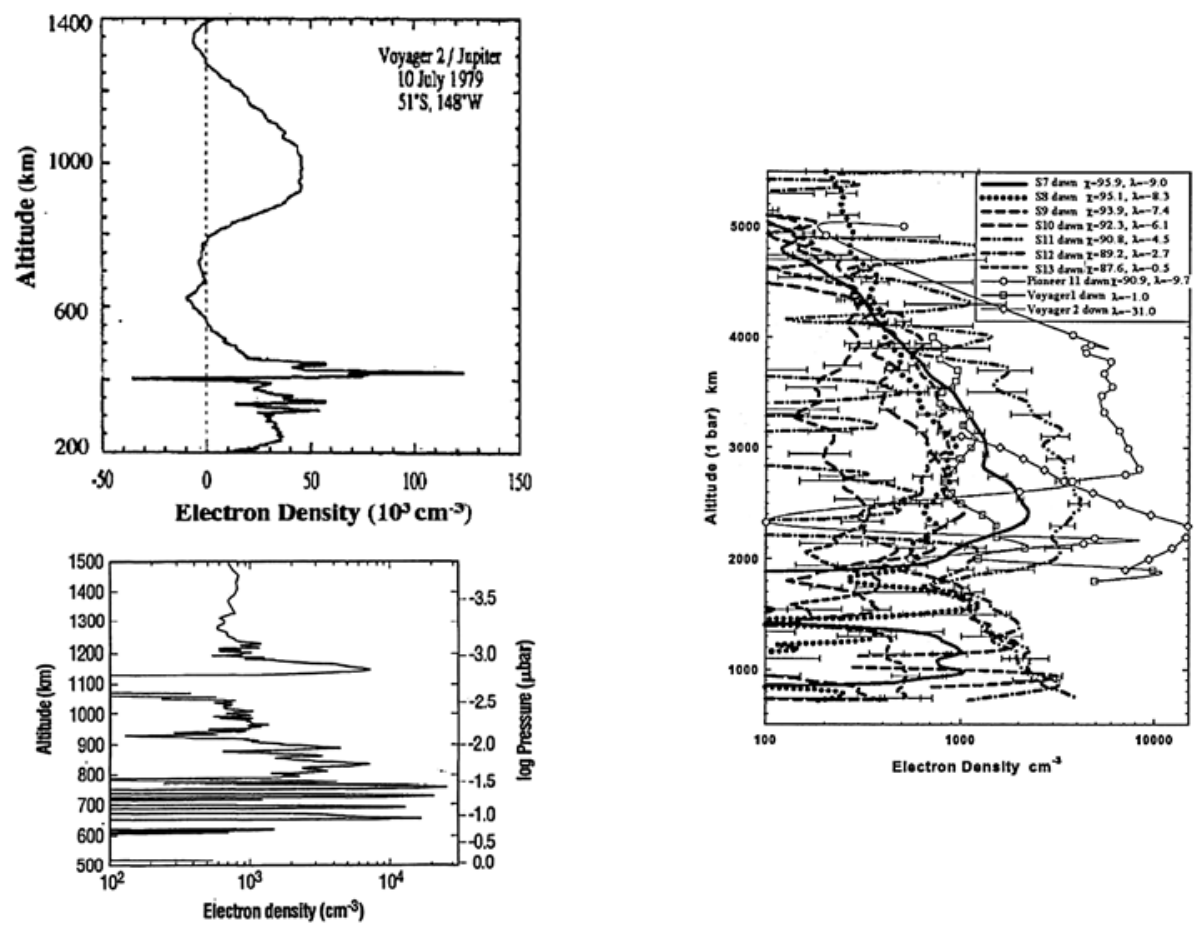

Fig. 3 Electron concentration profile at Jupiter (top-left) (Hinson et al. 1998), Neptune (lower-left) (From J.R. Lyons, Sience 268:648 (1995). Reprinted with permission from AAAS) and Saturn (right) (Nagy et al. 2006)

with a concentration of $(20-120) \times 10^{3} \mathrm{~cm}^{-3}$ that might be formed in response to vertical shear in the zonal wind or plasma instabilities. Pre-Voyager theoretical models of the lower ionosphere predicted a layer of hydrocarbon ions in the $300-400 \mathrm{~km}$ altitude range (Kim and Fox 1994); although the calculated magnitude is around two order of magnitude smaller than the observed one. The difference between theory and observations seems too large to be reconciled by considering atmospheric processes. A plausible explanation is that the lowest layer is composed of long-lived metallic ions supplied by meteoroids or by the Galilean satellites (Hinson et al. 1998).

\subsection{Saturn}

Saturn presents quite a complex ionosphere where several ionization sources and physical processes take place. In addition to the solar and cosmic radiation, water inflow and particle impact have important influences on the ionospheric structure. The lower part also presents some layered structure, as it has been detected by Cassini (Nagy et al. 2006). Figure 3 shows the electron concentration profile for exit (dawn terminator) measured by Pioneer 11, Voyager 1 and 2, and Cassini. We can observe that only Cassini was able to determine the structure of the lower part of the ionosphere. The peak densities are, in general, larger for the dusk results than for the dawn ones. The profile corresponding to the dawn terminator presents a thick layer in the $900-1500 \mathrm{~km}$ range, well below the main peak placed at around $2500 \mathrm{~km}$. For the dusk terminator, the layer is more sharp and could be partially included in 
the upper main ionosphere. The magnitude of the lower ionospheric layer is, in both cases, of around $1000 \mathrm{~cm}^{-3}$.

\subsection{Neptune}

Electron number density profile in Neptune observed during Voyager 2 occultation revealed sharp layers in the lower ionosphere with densities of around $10^{4} \mathrm{~cm}^{-3}$ (Lyons 1995), see Fig. 3. The magnitude of the layers are even higher than the upper peak. It must be taken into account that the uncertainty in the electron abundance is high in the lower ionosphere, by as much as a factor of two, but the altitude of the layers is well determined from the phase of the received signal. A simple explanation for these layers is that the long-lived metallic ions are compressed by a horizontal wind with a vertical shear acting on the ions in the presence of a magnetic field (Lyons 1995).

\section{The Interaction of Meteoroids with a Planetary Atmosphere}

In parallel to the experimental observations, many theoretical studies have considered the effect of dust in the atmosphere of Venus (McAuliffe and Christou 2006), Mars (Adolfsson et al. 1996; Pesnell and Grebowsky 2000; Molina-Cuberos et al. 2003), Jupiter (Grebowsky 1981; Hinson et al. 1998; Kim et al. 2001), Saturn (Moses and Bass 2000), Titan (Ip 1990; English et al. 1996; Molina-Cuberos et al. 2001), Neptune (Moses 1992; Lyons 1995) and Triton (Pesnell et al. 2004).

The evaluation of meteoroid effects on planetary atmospheres requires the knowledge of the mass and velocity distributions of the meteoroid flux through the Solar System. Then the dynamical evolution of small particles through the atmosphere has to be calculated in order to determine the deposition profiles of neutrals and ions. The concentration of each metallic compound is calculated by solving the continuity and momentum equations.

\subsection{Interplanetary Flux}

Collisions between asteroidal parent bodies or grains released by comets are the major source of meteoroids in the Solar System (Liou et al. 1995; Gurnett et al. 1997). Exogenous sources also exist, particles coming from the local interstellar medium cross the Solar System on hyperbolic orbits (Grün et al. 1993). The experimental information of the dust distribution beyond the orbit of the Earth comes from the dust detectors on board of Pioneer 10 and 11, Ulysses, Galileo and Cassini (Humes 1980; Grün et al. 1993; Altobelli et al. 2007):

The measurements by the penetration detector of Pioneer 10 indicate that the spatial density of $10^{-9} \mathrm{~g}$ meteoroids is essentially constant between 1 and $18 \mathrm{AU}$ (Humes 1980). The data obtained by the detector on Pioneer 11 show that meteoroids between 4 and $5 \mathrm{AU}$ are not in circular orbits near the ecliptic plane, but they follow randomly inclined orbits of high eccentricity (Humes 1980), which implies a cometary origin. During the Saturn encounters, the on board detectors measured an increase in the flux of about three orders of magnitude, probably as a result of impacts from ring particles (Humes 1980). The data obtained with the penetration detector on board Pioneer 10 and 11 have similar shapes in spite of the differences on the threshold mass, $10^{-9}$ and $10^{-8} \mathrm{~g}$, respectively, which means that the particle size distribution does not change strongly with the orbital radius (Cuzzi and Estrada 1998). 
The high sensitivity Cassini Dust Analyzer (Altobelli et al. 2007) measured the dust particles between Jupiter and Saturn and found two main group. The first group of impacts consists of particles on bound and prograde orbits coming from the dust ram direction, most probably on low eccentric and low inclined orbits, and they show a large spread in mass. The possible sources are short-period Jupiter family comets or circumsolar dust. Impactors of the second group were identified as interstellar dust particles, perhaps including a minority of beta-meteoroids. The upper limit value of the flux and the particles size are in very good agreement with what is expected from the Ulysses data (Grün et al. 1993) and model predictions.

The dust environment in the outer Solar System has been sounded by the Voyagers. The plasma wave instruments were able to detect a small but persistent level of dust impacts (up to $51 \mathrm{AU}$ for Voyager 1 and up to $33 \mathrm{AU}$ for voyager 2). The average number density obtained is estimated to be around $2 \times 10^{-8} \mathrm{~m}^{-3}$, and the average mass of around $10^{-11} \mathrm{~g}$ (Gurnett et al. 1997). The ecliptic latitudes of the paths taken by Voyager 1 and 2 were quite different. After the flyby of Saturn at 9.5 AU, Voyager proceeded northward from the ecliptic plane at an asymptotic ecliptic latitude of about $35^{\circ}$. Voyager 2 remained very close to the ecliptic plane until the flyby of Neptune at 30 AU. Considering the differences in the spacecraft trajectories, the observed variations in number densities were small and (Gurnett et al. 1997) concluded that comets are the most likely source for interplanetary dust particles in the outer Solar System.

\subsection{Entry Velocity}

For particles on bound orbits, the velocity distribution of dust particles arriving a planet depends on the distance to the Sun and on the planetary gravitational field. The velocity decreases with the distance to the Sun. Cuzzi and Estrada (1998) found the relationship between the orbital velocity of the meteoroids and the distance to the Sun to be:

$$
v(R)=\frac{v_{1}}{\sqrt{R_{A U}}},
$$

where $v_{1}$ is the velocity at $1 \mathrm{AU}$ and $R_{A U}$ the distance to the Sun.

Meteoroids penetrate the atmosphere at higher velocities than predicted by (1). The planetary gravitational field accelerates the particle and its orbit becomes parabolic. If $v^{*}$ is the relative speed of a particle with respect to the planet, then the meteoroid speed $v(r)$ at a distance $r$ from the planetary centre is:

$$
v(r)=\sqrt{v_{\mathrm{esc}}^{2}(r)+v^{* 2}}
$$

where $v_{\text {esc }}(r)$ is the planetary escape velocity of an object at a distance $r$.

The gravitational field also produces an enhancement in the cross section of a planet and, therefore, an increase in the meteoroid flux by a factor (Bauer 1973):

$$
G=1+\left(\frac{v_{\mathrm{esc}}}{v^{*}}\right)^{2}
$$

Table 1 shows the characteristic velocities of meteoroids arriving at Solar System bodies with a noticeable atmosphere. $V_{\min }$ and $V_{\max }$ represent the minimum and maximum velocities reaching the top of the atmosphere, respectively, where meteoroids in heliocentric orbits are assumed. The minimum velocity corresponds to the planetary escape velocity at the top 
Table 1 Characteristic velocities $\left(\mathrm{km} \mathrm{s}^{-1}\right)$

The case of Titan includes the gravitational focus of Saturn, $V_{\text {orb }}$ is the orbital velocity around Saturn

\begin{tabular}{|c|c|c|c|c|}
\hline & $V_{\min }$ & $V_{\odot}$ & $V_{\text {orb }}$ & $V_{\max }$ \\
\hline Venus & 10 & 50 & 35 & 85 \\
\hline Earth & 11 & 42 & 30 & 72 \\
\hline Mars & 5 & 34 & 24 & 58 \\
\hline Jupiter & 60 & 19 & 13 & 69 \\
\hline Saturn & 35.5 & 13.7 & 9.7 & 42.5 \\
\hline $\operatorname{Titan}^{\mathrm{d}}$ & 2.6 & 13.7 & 5.58 & 29.1 \\
\hline Uranus & 21 & 9.6 & 6.8 & 26.6 \\
\hline Neptune & 23.5 & 7.7 & 5.5 & 26.9 \\
\hline
\end{tabular}

of the atmosphere. The maximum velocity corresponds to a particle with the solar system scape velocity $V_{\odot}$ orbiting the Sun in a retrograde orbit. $V_{\text {orb }}$ is the planetary orbital velocity.

It can be observed that the range of velocities $\left(V_{\max }-V_{\min }\right)$ is very wide for terrestrial planets due to the combined effect of a low escape velocity and high orbital velocity. For the giant planets this velocity range is much smaller.

\subsection{Meteoroid Ablation}

The problem of determining the physical evolution of a particle penetrating the atmosphere was first treated in detail by Öpik (1958). Here we briefly describe the processes describing the loss of velocity and mass of a spherical small particle, based in the work of Lebedinets et al. (1973). We do not consider aspects like fragmentation, non spherical shape, mixed compositions or differential ablation which are usually important for the detailed modeling of the Terrestrial atmosphere or for high mass particles.

Meteoroids penetrating the atmosphere are accelerated by the planetary gravitational field and slowed down by collisions with atmospheric constituents. Collisions also remove part of the mass and heat the particle surface producing an additional loss of mass by evaporation. The increase of the particles' temperature by atmospheric collisions is balanced with thermal radiation and loss of heat through ablation.

The dynamical evolution of a small particle penetrating the atmosphere is calculated by solving the motion, ablation and energy equations. The motion equation relates the decrease in relative impact velocity $v$ due to the drag of the atmosphere:

$$
\cos \theta \frac{d v}{d z}=\frac{\Gamma A \rho v}{\delta^{2 / 3} m^{1 / 3}}
$$

where $\theta$ is the entry angle, and $v, m$ and $\delta$ are the meteoroid velocity, mass and density, respectively. The atmospheric drag depends on the drag coefficient, $\Gamma$, the atmospheric mass density, $\rho$, and on the meteoroid shape, through factor $A$.

The ablation equation relates the loss of mass $m$ from a meteoroid due to evaporation and sputtering:

$$
\cos \theta \frac{d m}{d z}=-\frac{4 A K_{1} m^{2 / 3}}{\delta^{2 / 3} v T^{1 / 2}} e^{-K_{2} / T}-\frac{\Lambda_{S} A \rho m^{2 / 3} v^{2}}{2 Q^{2 / 3}}
$$

where $K_{1}$ and $K_{2}$ are constants describing the dependence of the evaporation rate on temperature, $T . \Lambda_{S}$ is the sputtering coefficient and $Q$ the energy of evaporation of $1 \mathrm{~g}$ of the meteoroid. 
Finally, the energy equation provides the thermal evolution of the particle as a function of the increase of temperature due to the heating by sputtering, thermal radiation from the body surface and the deposition of energy by evaporation:

$$
\cos \theta \frac{d T}{d z}=\frac{4 A \rho v^{2}}{8 C \delta^{2 / 3} m^{1 / 3}}\left(\Lambda-\Lambda_{S}\right)-\frac{4 A \sigma T^{4}}{C \delta^{2 / 3} v m^{1 / 3}}-\frac{4 A K_{1} Q}{C \delta^{2 / 3} T^{1 / 2} m^{1 / 3} v} e^{-K_{2} / T}
$$

where $\Lambda$ the heat transfer coefficient, $\sigma$ the Stefan-Boltzmann constant and $C$ the heat capacity of the meteoroid substance.

The energy, mass and momentum are couple by a system of equations which usually demand a high precision numerical method and a small discretization grid in order to solve it. In principle, the effect of a velocity distribution has to be taken into account in the modeling, mainly if a high precision of the meteoroid mass deposition profile is required, as usually occurs to the Earth case. However, most of the numerical models of the extraterrestrial atmospheres simply consider a monochromatic distribution at the mean velocity, rather than a distribution of velocities, and analyse the effect of different incoming velocities. For low gravity planets a mean angle of 45 degrees for incoming particles can be used. The effect of non-vertical entry is to elevate the altitude at which ablation occurs. For massive planets the gravitational focus shifts the distribution of incident angle towards vertical.

Icy meteoroids coming from comets ablate more easily and at higher altitude than stony meteoroids, which are produced in the asteroid belt. Rocky material introduces a higher amount of metal constituents to the atmosphere than icy meteoroids.

Once the ablation of meteoroids is known, the linear concentration of the individual ion species can be calculated by

$$
\alpha_{i}=-\frac{p_{i} \beta_{i}}{m_{i} v} \frac{d m}{d t}
$$

where $p_{i}$ is the ratio of atom $i$ type to the total, $m_{i}$ is the atomic mass, and $\beta_{i}$ is the ionization probability, which depends on the ion produced and meteoroid velocity. For low velocity $\leq 35 \mathrm{~km} \mathrm{~s}^{-1}$, for which no secondary ionization or recombination take place, Jones (1997) proposed an empirical expression:

$$
\beta_{i}=k_{i}\left(v-v_{i}\right)^{2} v^{0.8}
$$

where $k_{i}$ is an experimental value, which depends on the element and $v_{i}$ is a cut off velocity, For high velocity particles, a more general expression can be used (Lebedinets et al. 1973):

$$
\beta_{i}=C v^{7 / 2} .
$$

The production rate of $i$ type ions $\left(P_{i}\right)$ is calculated as:

$$
P_{i}=\int \alpha_{i} f(m) d m
$$

where $f(m)$ is the flux per unit of micrometeoroidal mass.

\section{Modelling Metallic Layers in Planetary Atmospheres}

The meteoroid mass deposition profiles are calculated by adapting the flux of interplanetary dust and solving the dynamical evolution of the particles, as described in the above section. 

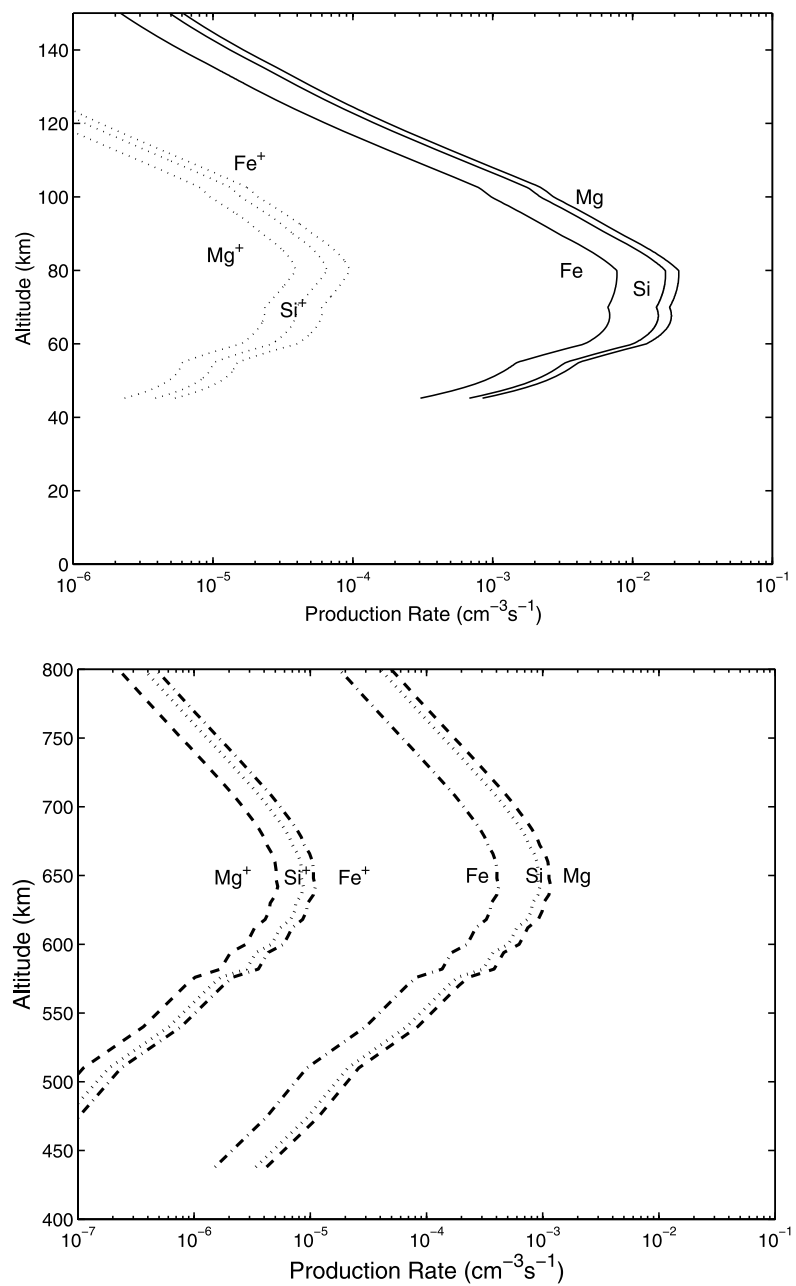

Fig. 4 Neutral and ionic deposition rates of $\mathrm{Mg}, \mathrm{Fe}$ and $\mathrm{Si}$ due to the ablation of meteoroids with entry velocity of $18 \mathrm{~km} \mathrm{~s}^{-1}$ in the atmosphere of Mars (top) (Molina-Cuberos et al. 2003) and Titan (bottom) (adapted from Molina-Cuberos et al. 2001) to consider the same meteoroid composition than on Mars

We have used the model by Grün et al. (1985), which assumes an isotropic flux of meteoroids at Earth's orbit with effective density of $2.5 \mathrm{~g} \mathrm{~cm}^{-3}$ and mean velocity of $v(1 \mathrm{AU})=$ $20 \mathrm{~km} \mathrm{~s}^{-1}$. The model considers mass ranging from $10^{-18}$ to $100 \mathrm{~g}$, although the main contribution to the total mass is due to particles ranging from $10^{-7}$ to $10^{-4} \mathrm{~g}$.

Figure 4 shows the neutral and ion deposition rate of magnesium, iron and silicon in the atmosphere of Mars (top) and Titan (bottom), assuming the interplanetary dust is mainly composed of carbonaceous chondrites, that have a relative concentration of $\mathrm{Mg}=6.1 \%$, $\mathrm{Si}=5.7 \%$ and $\mathrm{Fe}=5.1 \%$ (Anders and Ebihara 1982).

Table 2 shows the altitude range where the maximum of the meteoroid ablation occurs and the altitude of the ionospheric layers that could consists of metallic ions. Please note that the ionospheric peak is located in all the cases quite close to the ablation altitude. 
Table 2 Theoretical predictions of the altitude of the meteoroid deposition peak and altitude of ionospheric layer that could consist on metallic ions. $\odot$ represents ice meteoroids and $\bullet$ silicate ones

\begin{tabular}{llll}
\hline Planet & $\begin{array}{l}\text { Ablation } \\
(\mathrm{km})\end{array}$ & Reference & $\begin{array}{l}\text { Ionospheric } \\
\text { peak }(\mathrm{km})\end{array}$ \\
\hline Venus & $110-120$ & This work & $120-130 \mathrm{~km}$ \\
Earth & $85-95$ & McNeil et al. 1998 & $90-100 \mathrm{~km}$ \\
Mars & $75-85$ & Molina-Cuberos et al. 2003 & $65-100$ \\
Jupiter & $300-400$ & Kim et al. 2001 & $300-550$ \\
Saturn & $790-1290 \odot$ & Moses and Bass 2000 & $900-1200$ \\
& $610-790 \bullet$ & Moses and Bass 2000 & \\
Titan & $650-700$ & Molina-Cuberos et al. 2001 & No evidences \\
Neptune & $500-800 \bullet$ & Moses 1992 & $600-1000 \mathrm{~km}$ \\
& $250-500 \odot$ & Moses 1992 & \\
\hline
\end{tabular}

The altitude of the ablation depends on the physical characteristics of meteoroids (volatility, speed, size and composition). The radio-occultation measurements of ionospheric layers of long-lived metallic ions through the Solar System planets could, therefore, provide information about properties and composition of interplanetary dust. The distribution of metals is, however, affected by transport and layering.

Once the meteoroid deposition in the atmosphere is known, the concentration of each neutral and ion species is calculated from the continuity and momentum equations that, assuming steady state, may be expressed as:

$$
\begin{aligned}
P_{i}-n_{i} l_{i}= & \frac{\partial}{\partial z} n_{i} v_{i} \\
v_{i}= & -D_{i}\left(\frac{1}{n_{i}} \frac{\partial n_{i}}{\partial z}+\frac{1}{H_{i}}+\frac{1}{T} \frac{\partial T}{\partial z}\right) \\
& -K\left(\frac{1}{n_{i}} \frac{\partial n_{i}}{\partial z}+\frac{1}{H}+\frac{1}{T} \frac{\partial T}{\partial z}\right)
\end{aligned}
$$

where $i$ denotes the $i$ th constituent, $z$ the altitude, $n_{i}$ the concentration, $P_{i}$ the production, $l_{i}$ the specific loss, $T$ the temperature. $v_{i}$ is the mean vertical velocity, $D_{i}$ and $K_{i}$ are molecular and eddy diffusion coefficients, $H_{i}$ and $H$ are the individual and atmospheric scale heights.

Vertical transport of metallic species is mainly produced by turbulent and molecular diffusion, the former being more effective at lower levels. Diffusion theory provide analytical expressions for the molecular diffusion coefficients $D_{i}$ (Chapman and Cowling 1970). The turbulent diffusion is the least known factor in the modeling of an atmosphere. It is typically parameterized by means of an eddy diffusion coefficient $K$.

The production of metallic ions depends on the atmospheric and ionospheric characteristics. Other ionization sources, like solar radiation or electrons, provide atmospheric ions that transfer the charge to metallic atoms by charge exchange reactions, which is a very important source for metallic ions. In addition, the photoionization of metallic neutrals increases the production of metallic ions. 


\subsection{Earth}

Meteoric metals in the terrestrial atmosphere have been modeled in detail. The chemistry and temporal and spatial variations have been extensively treated in the literature (Swider 1969; Aikin and Goldbert 1973; Carter and Forbes 1999; Joiner and Aikin 1996). In particular, special attention has been paid to the most abundant metallic species, magnesium (McNeil et al. 1996; Plane and Helmer 1995), iron (Helmer et al. 1998; Carter and Forbes 1999), silicon (Kopp et al. 1995), potassium (Eska et al. 1999), sodium and calcium (McNeil et al. 1998; Plane et al. 1999) and the chemistry is well known.

Metals consititute a very small fraction of the total atmospheric constituents in the Eregion; however the ionized fraction of metallic atoms is very high when compared with other atmospheric compounds. The reason for the relatively high concentration of metallic ions is their low electron recombination rate, several order of magnitude slower than the recombination of the most abundant ambient ions, $\mathrm{O}_{2}^{+}$and $\mathrm{NO}^{+}$. The chemical lifetime of metallic ions is very long and their vertical distribution is strongly influenced by transport mechanisms such as eddy diffusion and layering due to wind shears and electric field. Metallic species are removed from the $E$-layer by downward transport. Three-body association reactions with atmospheric neutrals produce molecules in the gas phase that subsequently condensate and coagulate to aggregates and aerosols (Hungen et al. 1980). Figure 6 shows a schematic diagram of reactions involving magnesium species, as an example of the chemistry of metallic compounds in the terrestrial atmosphere. It was developed from the works by Plane and Helmer (1995), McNeil et al. (1996) and McNeil et al. (1998).

Magnesium ion are mainly produced by charge exchange with atmospheric ambient species $\left(\mathrm{O}_{2}^{+}, \mathrm{NO}^{+}\right.$and $\left.\mathrm{O}^{+}\right)($Grebowsky et al. 1998), and also by direct meteoric ionization and photoionization. The recombination of $\mathrm{Mg}^{+}$with electrons is not the main loss process of $\mathrm{Mg}$ ions. Three body reactions of $\mathrm{Mg}^{+}$with $\mathrm{O}_{2}$ and $\mathrm{N}_{2}$ produce $\mathrm{MgO}_{2}^{+}$(Plane and Helmer 1995) and $\mathrm{MgN}_{2}^{+}$(McNeil et al. 1996) and two body reaction with ozone leads to the formation of $\mathrm{MgO}^{+}$. These ions are recycled to neutral $\mathrm{Mg}$ through molecular dissociative recombination. The chemistry of neutral magnesium is determined by two/three body reactions with oxygen species $\left(\mathrm{O}, \mathrm{O}_{2}\right.$ and $\left.\mathrm{O}_{3}\right)$, which produce $\mathrm{MgO}$ and $\mathrm{MgO}_{2}$. The final sink of $\mathrm{Mg}$ may be $\mathrm{Mg}(\mathrm{OH})_{2}$ as obtained by Plane and Helmer (1995) or $\mathrm{MgCO}_{3}$ as calculated McNeil et al. (1996).

Grebowsky et al. (1998) compiled all published studies describing rocket flights which measured meteoric ions between 1963 and 1991 . They found that the observed $\mathrm{Mg}^{+}$concentrations are lower than those yielded by models and they also confirmed that meteor showers do have significant impact on the average ionospheric composition.

\subsection{Mars}

Terrestrial knowledge is the starting point to model the meteoroid effects on the atmosphere of Mars. The chemistry of metallic ions is quite similar to the terrestrial case, $\mathrm{CO}_{2}$ playing the role of the third body in three-body reactions in Mars instead of $\mathrm{N}_{2}$ on Earth.

Magnesium and iron ions are produced by direct meteoric ionization, photoionization and charge exchange with atmospheric ions, mainly $\mathrm{O}_{2}^{+}$. The last one is the main source for production of metallic ions. Magnesium and iron follow quite similar processes. The oxidation by ozone is the most efficient mode of converting atomic metals into neutral oxides. Once $\mathrm{MgO}$ and $\mathrm{FeO}$ are formed, three body association of $\mathrm{CO}_{2}$ provides carbonated metallic atoms, which are the more stable neutrals. Metallic ions can undergo electron recombination or be converted to oxygenated ions. At higher pressure, molecular association by three-body 


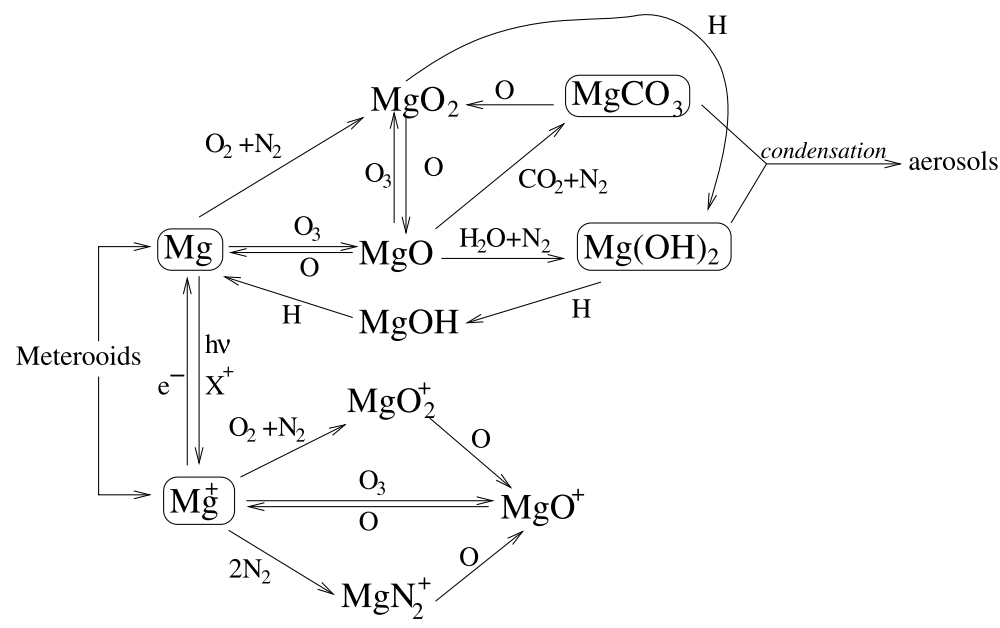

Fig. 5 Schematic diagram of the chemistry of magnesium species in the terrestrial atmosphere, where $\mathrm{X}^{+}$ represents a non-metallic ion (mainly $\mathrm{O}^{+}$and $\mathrm{NO}^{+}$) and $\mathrm{h} v$ photoionization. Adapted from Plane and Helmer (1995), McNeil et al. (1996, 1998)

Fig. 6 Schematic diagram of the chemistry of iron species in the martian atmosphere, where $\mathrm{X}^{+}$ represents a non-metallic ion and $\mathrm{h} v$ photoionization. Adapted from Molina-Cuberos et al. (2003)

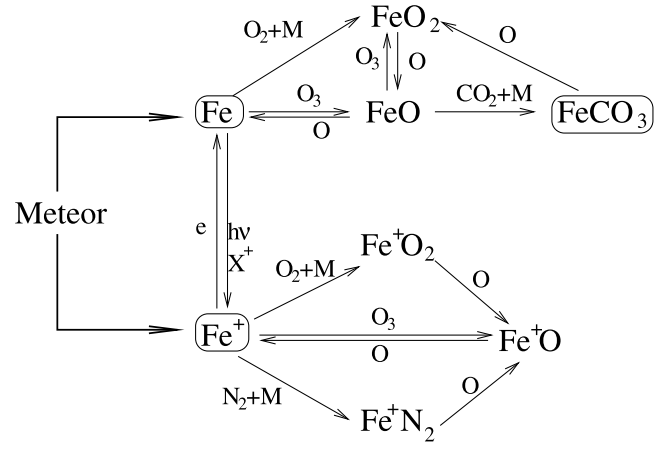

reactions produce molecular ions, which undergo molecular dissociative recombination to form $\mathrm{Fe}$ and $\mathrm{Mg}$ (Molina-Cuberos et al. 2003). Figure 6 shows a schematic diagram of the chemistry of iron species. For the case of $\mathrm{Mg}$, it follows a general scheme similar to Fe, but with different rates.

Pesnell and Grebowsky (2000) modeled the effect of magnesium in the atmosphere of Mars and predicted a persistent layer of $\mathrm{Mg}^{+}$in the order of $10^{4} \mathrm{~cm}^{-3}$ at around $80 \mathrm{~km}$, which is a factor of around 20 times lower than the main ionospheric peak placed at $130 \mathrm{~km}$. Molina-Cuberos et al. (2003) developed daytime and nighttime models for iron and magnesium produced by meteoric ablation, and the effect of solar activity, and seasonal variations was also explored. They found a meteoric layer formed by $\mathrm{Fe}^{+}$and $\mathrm{Mg}^{+}$with a magnitude of the order of $10^{4} \mathrm{~cm}^{-3}$ at noon and decreases by two orders of magnitude during the night. The agreement between the model and the daytime measurements taken by Mars Express (Pätzold et al. 2005) some years later is quite good. Figure 7 shows (solid lines) the electron concentration predicted and a pair of experimental profiles that match better with the theoretical model. 
Fig. 7 Theoretical predictions (Molina-Cuberos et al. 2003) (lines) and experimental determination (Pätzold et al. 2005) of meteoroid layers in the Martian ionosphere

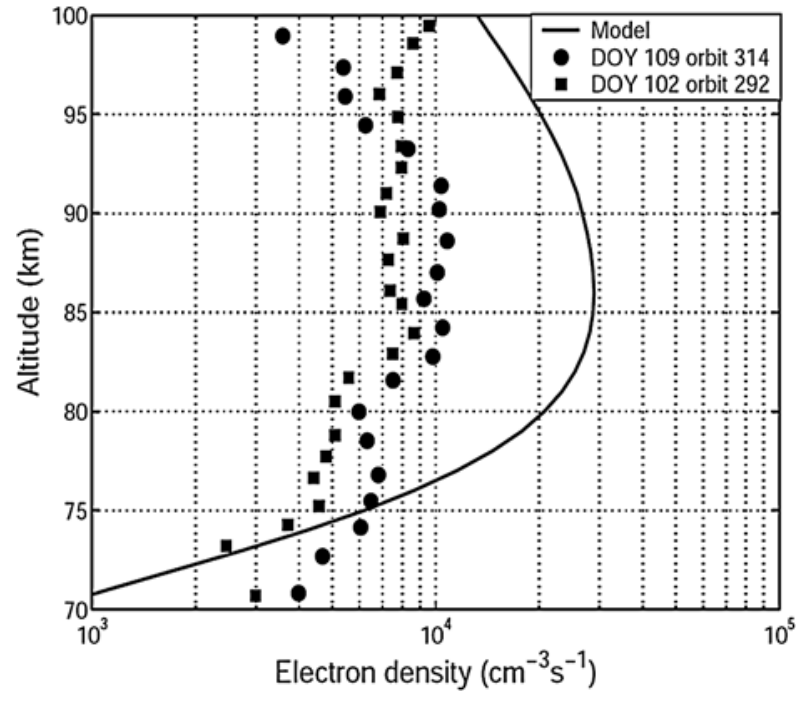

Fig. 8 Schematic diagram of the chemistry of metals in the ionosphere of giant planets, where $\mathrm{M}$ represents $\mathrm{Mg}^{+}$or $\mathrm{Fe}^{+}$. Electron recombination of metals have not been included for higher clarity, adapted from Kim et al. (2001)

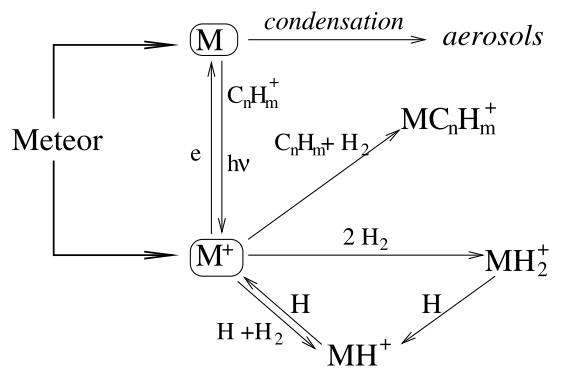

\subsection{Giant Planets}

The hydrogen-hydrocarbons atmosphere of the giant planets is more difficult to model than the Martian one, due to the lack of measurements of reaction rates between hydrocarbons and metallic ions.

The ionospheres of gaseous planets are usually dominated by $\mathrm{H}^{+}$in the upper part and $\mathrm{H}_{3}^{+}$prevails below (Kim et al. 2001; Moses and Bass 2000). If metals are not considered in the modeling, then hydrocarbons are the major ionic species in the lower ionosphere (Kim and Fox 1994). However the predicted densities are much lower than the observed ones. The inclusion of meteoric ablation into the atmosphere that produces metal ions that take the place of hydrocarbons as the major ionic species in the lower part of the ionosphere (Kim et al. 2001; Moses and Bass 2000; Lyons 1995). Figure 8 shows a short scheme of the chemistry of metals in the atmosphere of giant planets.

The high gravitational focus of giant planets and the long distance to the Sun results in metallic ions that are mainly produced by charge exchange with atmospheric ions and direct ionization from ablation; the other source, photoionization of metallic neutrals, is much less important. Metallic ions are removed by three-body reactions with hydrocarbons and hydrogen, and the metallic atoms are lost by condensing onto existing aerosols or dust particles. The depletion of atoms is parameterized by assuming a constant lifetime, which depends on the number of dust particles and their size; typical values in the range of (1-6) 
$\times 10^{5} \mathrm{~s}$ have been used in the modeling of Jupiter (Kim et al. 2001), Saturn (Moses and Bass 2000), and Neptune (Lyons 1995). Three-body reaction between metallic ions $\left(\mathrm{Fe}^{+}, \mathrm{Mg}^{+}\right)$ with the most abundant atmospheric neutral $\left(\mathrm{H}_{2}\right)$ has not been confirmed in the laboratory. Even by assuming that the reaction occurs, it results in little net loss of ions, due to the adduct $\left(\mathrm{Fe}^{+} \cdot \mathrm{H}_{2}\right.$ or $\left.\mathrm{Mg}^{+} \cdot \mathrm{H}_{2}\right)$ undergoing a sequence of reactions with $\mathrm{H}$ atoms which restore metallic ions in the Jovian atmosphere (Kim et al. 2001).

The electron concentration profile of outer planets shows frequent gaps, specially at the dawn terminators, see Fig 3. The layering that is frequently observed in the lower ionospheres of the outer planets could be caused by long-lived atomic ions being moved by horizontal winds that possess vertical shear, such as might occur with atmospheric tides and gravity waves interacting with meteoric ions.

The ablation of meteoroid in the Jovian atmosphere and the chemistry of meteoric ions $\left(\mathrm{O}^{+}, \mathrm{C}^{+}, \mathrm{Si}^{+}, \mathrm{Fe}^{+}, \mathrm{Mg}^{+}, \mathrm{Na}^{+}\right.$and $\left.\mathrm{S}^{+}\right)$was modeled by Kim et al. (2001). They found a layer of meteoric ions in the altitude region of $350-450 \mathrm{~km}$, above the 1-bar level, with a peak total ion concentration of several times $10^{4} \mathrm{~cm}^{-3}$, which are comparable with the observed one. Moses and Bass (2000) studied the effects of external material on the chemistry and structure of Saturn's ionosphere, they found that the ionospheric structure is dominated by two major peak, with $\mathrm{H}^{+}$creating the high-altitude peak and $\mathrm{Mg}^{+}$, representing the metallic ions, the low-altitude peak with a magnitude in the order of $10^{4} \mathrm{~cm}^{-3}$, similar to the upper one. Lyons (1995) also considered metal ions to model the lower ionosphere of Neptune and calculated a concentration of $\mathrm{Mg}^{+}$around $1000 \mathrm{~cm}^{-3}$, one order of magnitude lower than the one observed by Voyager 2. The differences are reduced if the magnesium ions are compressed into sharp layers by a sinusoidal vertical wind.

\subsection{Titan}

The Voyager 1 fly-by of Titan was able to determine an ionospheric peak of $2400 \pm$ $1100 \mathrm{~cm}^{-3}$ at $1180 \pm 150 \mathrm{~km}$ (Bird et al. 1997), and did not provide any information from below the peak. Therefore, Voyager did not show any evidence for meteoroids effects in its atmosphere. From 2004, Cassini has been orbiting Saturn and several opportunities to sound the ionosphere by radio-occultation and even to determine ionic mass by INMS (Ion and Neutral Mass Spectrometer) will be available in the near future. In spite of the lack of experimental observations of the effects of meteoroids at Titan, some theoretical models have investigated the effects of meteoroids in the composition of neutral (English et al. 1996) and ion (Molina-Cuberos et al. 2001) species. Molina-Cuberos et al. (2001) investigated the ablation of meteoroids and found that long-lived metallic ions considerably change the predictions of the electron number density due to models which only consider solar radiation and electrons trapped in the magnetosphere of Saturn. By using a simple model where metallic ions are lost by termolecular associations with neutral molecules, they concluded that an ionospheric layer could be present at around $700 \mathrm{~km}$, with an electron concentration peak similar in magnitude to the one produced by solar radiation. Petrie (2004) has developed theoretical calculations of $\mathrm{Mg}^{+}$reactions with the atmospheric compounds of Titan $\left(\mathrm{N}_{2}, \mathrm{CH}_{4}\right.$ and some nitrogenated hydrocarbons), which allowed the magnesium chemistry to be modelled in more detail an predicted the radical $\mathrm{MgNC}$ as the final product.

Acknowledgements This work was supported by contracts ESP2003-00357. The authors thanks Ernesto Martín and Ann and Harry Callaway for their assistance in writing this paper. 


\section{References}

L.G. Adolfsson, B.A.S. Gustafson, C.D. Murray, The Martian atmosphere as a meteoroid detector. Icarus 119, 144-152 (1996)

A.C. Aikin, R.A. Goldbert, Metallic ions in the equatorial ionosphere. J. Geophys. Res. 78, 734-745 (1973)

A.C. Aikin, W.C. Maguire, Detection in the infrared of $\mathrm{Mg}^{+} \cdot \mathrm{CO}_{2}$ ion produced via meteoritic material in the Martian atmosphere. Bull. AAS 37, 1567 (2005)

N. Altobelli, V. Dikarev, S. Kempf et al., Cassini/Cosmic Dust Analyzer in situ dust measurements between Jupiter and Saturn. J. Geophys. Res. 112 (2007)

E. Anders, M. Ebihara, Solar-system abundances of the elements. Geochemica et Cosmochimica Acta 46, 2363-2380 (1982)

S.J. Bauer, Physics of Planetary Ionosphere (Spinger, Berlin, 1973)

M.K. Bird, R. Dutta-Roy, S.W. Asmar, T.A. Rebold, Detection of Titan's ionosphere from Voyager 1 radio occultation observations. Icarus 130, 426-436 (1997)

L.N. Carter, J.M. Forbes, Global transport and localized layering of metallic ions in the upper atmosphere. Ann. Geophysicae 17, 190-209 (1999)

S. Chapman, Notes on atmospheric sodium. Astrophys. J. 90, 309-316 (1938)

S. Chapman, T.G. Cowling, The Mathematical Theory of Non-Uniform Gases, 3rd edn. (Cambridge University Press, New York, 1970)

J.N. Cuzzi, P.R. Estrada, Compositional evolution of Saturn's rings due to meteoroid bombardment. Icarus 132, 1-35 (1998)

M.A. English, L.M. Lara, R.D. Lorenz, P. Ratcliff, R. Rodrigo, Ablation and chemistry of meteoric materials in the atmosphere of Titan. Adv. Space Res. 17, 157-160 (1996)

V. Eska, U. von Zahn, J.M.C. Plane, The terrestrial potassium layer $(75-110 \mathrm{~km})$ between $71 \mathrm{~S}$ and $54 \mathrm{~N}$ : Observations and modeling. J. Geophys. Res. 104(A8), 17173-17186 (1999)

G. Fjeldbo, W.C. Fjeldbo, V.R. Eshleman, Atmosphere of Mars: Mariner IV models compared. Science 153, $1518-1523$ (1966)

J.M. Grebowsky, Meteoric ion production near Jupiter. J. Geophys. Res. 86, 1537-1543 (1981)

J.M. Grebowsky, R.A. Goldberg, W.D. Pesnell, Do meteor showers significantly perturb the ionosphere? J. Atmospheric Sol.-Terr. Phys. 60, 607-615 (1998)

E. Grün, H.A. Zook, M. Baguhl et al., Discovery of Jovian dust streams and interstellar grains by the Ulysses spacecraft. Nature 362, 428-430 (1993)

E. Grün, H.A. Zook, H. Fechtig, R.H. Giese, Collisional balance of the meteoritic complex. Icarus 62, 244$272(1985)$

D.A. Gurnett, J.A. Ansher, W.S. Kurth, L.J. Granroth, Micron-sized dust particles detected in the outer solar system by the Voyager 1 and 2 plasma wave instruments. Geophys. Res. Lett. 24, 3125-3128 (1997)

H. Helmer, J.M.C. Plane, J. Qian, C.S. Gardner, A model of meteoric iron in the upper atmosphere. J. Geophys. Res. 103, 10,913-10,925 (1998)

D.P. Hinson, F.M. Flasar, A.J. Kliore et al., Jupiter's ionosphere: Results from the first Galileo radio occultation experiment. Geophys. Res. Lett. 24, 2107-2110 (1997)

D.P. Hinson, J.D. Twicken, E.T. Karayel, Jupiter's ionosphere: New results from Voyager 2 radio occultation measurements. J. Geophys. Res. 103, 9505-9520 (1998)

D.W. Hughes, Meteors, in Cosmic Dust, ed. by J.A.M. McDonnell (Wiley, Chichester, 1978), pp. 123-184

D.H. Humes, Results of Pioneer 10 and 11 meteoroid experiments: Interplanetary and Near-Saturn. J. Geophys. Res. 85, 5841-5852 (1980)

D.M. Hungen, R.P. Turco, O.B. Toon, Smoke and dust particles of meteoric origin in the mesosphere and stratosphere. J. Atmospheric Sci. 37, 1342-1357 (1980)

W.H. Ip, Meteoroid ablation processes in Titan's atmosphere. Nature 345, 511-512 (1990)

J. Joiner, A.C. Aikin, Temporal and spatial variations in the upper atmospheric $\mathrm{Mg}^{+}$. J. Geophys. Res. 101, 5239-5249 (1996)

W. Jones, Theoretical and observational determinations of the ionization coefficient of meteors. Mon. Not. R. Astron. Soc. 288, 995-1003 (1997)

Y.H. Kim, J.L. Fox, The chemistry of hydrocarbon ions in the Jovian ionosphere. Icarus 112, 310-325 (1994)

Y.H. Kim, W.D. Pesnell, J.M. Grebowsky, J.L. Fox, Meteoric ions in the ionosphere of Jupiter. Icarus 150, 261-278 (2001)

A.J. Kliore, I.R. Patel, A.F. Nagy et al., Initial observations of the nightside ionosphere of Venus from Pioneer Venus orbiter radio occultations. Science 205, 99-102 (1979)

E. Kopp, On the abundance of metal ions in the lower ionosphere. J. Geophys. Res. 102, 9667-9675 (1997)

E. Kopp, F. Balsiger, E. Murad, Silicon molecular ions in the D-region. Geophys. Res. Lett. 22, 3473-3476 (1995) 
V.N. Lebedinets, A.V. Manochina, V.B. Shushkova, Interaction of the lower thermosphere with the solid component of the interplanetary medium. Planet. Space Sci. 21, 1317-1332 (1973)

J.C. Liou, S.F. Dermott, Y.L. Xu, The contribution of cometary dust to the zodiacal cloud. Planet. Space Sci. 43, 717-722 (1995)

J.R. Lyons, Metal ions in the atmosphere of Neptune. Science 267, 648-651 (1995)

B. Mason, ed. by B. Mason, Gordon and Breach, Handbook of Elemental Abundances in Meteorites (New York, 1971)

J.P. McAuliffe, A.A. Christou, Modelling meteor ablation in the Venusian atmosphere. Icarus 180, 8-22 (2006)

W.J. McNeil, S.T. Lai, E. Murad, A model for meteoric magnesium in the ionosphere. J. Geophys. Res. 101, 5251-5229 (1996)

W.J. McNeil, S.T. Lai, E. Murad, Differential ablation of cosmic dust and implications for the relative abundances of atmospheric metals. J. Geophys. Res. 103, 10,899-10,911 (1998)

G.J. Molina-Cuberos, H. Lammer, W. Stumptner et al., Ionospheric layer induced by meteoric ionization in Titan's atmosphere, Planet. Space Sci. 49, 143-153 (2001)

G.J. Molina-Cuberos, O. Witasse, J.P. Lebreton, R. Rodrigo, J.J. López-Moreno, Meteoric ions in the atmosphere of Mars. Planet. Space Sci. 51, 239-249 (2003)

J.I. Moses, Meteoroid ablation in Neptune's atmosphere. Icarus 99, 268-383 (1992)

J.I. Moses, S.F. Bass, The effects of external material on the chemistry and structure of Saturn's ionosphere. J. Geophys. Res. 105, 7013-7052 (2000)

A.F. Nagy, A.J. Kliore, E. Marouf et al., First results from the ionospheric radio occultations of Saturn by the Cassini spacecraft. J. Geophys. Res. 111 (2006)

E.J. Öpik, Physics of Meteor Flight in the Atmosphere (Wiley, New York, 1958)

M. Pätzold, S. Tellmann, B. Häusle et al., A sporadic third layer in the ionosphere of Mars. Science 310, 837-839 (2005)

W.D. Pesnell, J. Grebowsky, Meteoric magnesium ions in the Martian atmosphere. J. Geophys. Res. 105, 1695-1707 (2000)

W.D. Pesnell, J.M. Grebowsky, A.L. and Weisman, Watching meteros on Triton. Icarus 169, 482-491 (2004)

$\mathrm{S}$. Petrie, Products of meteoric metal ion chemistry within planetary atmospheres $1 . \mathrm{Mg}^{+}$at Titan. Icarus 171, 199-209 (2004)

J.M.C. Plane, M. Helmer, Laboratory Study of the Reactions $\mathrm{Mg}+\mathrm{O}_{3}$ and $\mathrm{MgO}+\mathrm{O}_{3}$. Implications for the chemistry of magnesium in the upper ionosphere. Faraday Discuss 100, 411-430 (1995)

J.M.C. Plane, R.M. Cox, R.J. Rollason, Metallic layers in the mesopause lower thermosphere region. Adv. Space Res. 24, 1559-1570 (1999)

N.A. Savich, V.A. Samovol, M.B. Vasilyev et al., The nighttime ionosphere of Mars from Mars-4 and Mars-5 radio occultation dual-frequency measurements, in Solar-Wind Interaction with the Planets Mercury, Venus, and Mars, ed. by N.F. Ness. NASA SP-397 (1976), pp. 41-46

D.F. Strobel, R. Yelle, D.E. Shemansky, S.K. Atreya, The Upper Atmosphere of Uranus in Uranus (University of Arizona Press, Tucson, 1991), pp. 65-109

W.J. Swider, Processes for meteoric elements in the $E$ region. Planet. Space Sci. 17, 1233-1246 (1969) 